\title{
Magnetic Resonance Characteristics of Negative Polarons in Neat Poly(3-hexyl-thiophene)
}

\author{
Borovkov V. I., ${ }^{1,2,1}$ Shchegoleva L. N. ${ }^{3}$ \\ ${ }^{1}$ Voevodsky Institute of Chemical Kinetics and Combustion, SB RAS, 3, Institutskaya str., \\ 630090, Novosibirsk, Russia \\ ${ }^{2}$ Novosibirsk State University, 2, Pirogova str., Novosibirsk, 630090, Russia \\ ${ }^{3}$ Vorozhtsov Novosibirsk Institute of Organic Chemistry, SB RAS, 9, Akad. Lavrent'ev Ave., \\ Novosibirsk, 630090, Russia
}

Supporting Information

${ }^{1}$ Corresponding author. Email: borovkov@kinetics.nsc.ru 


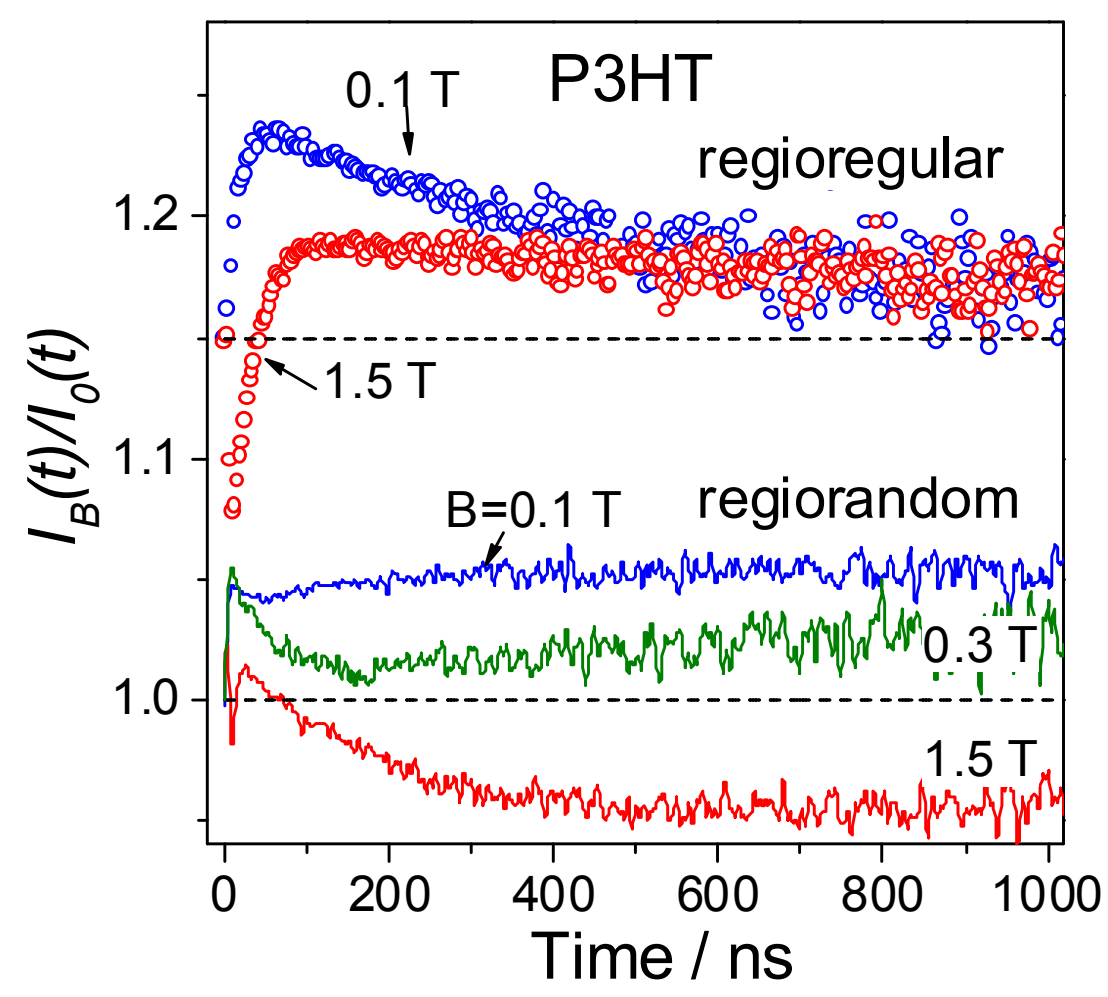

Figure S1. Ratios, $I_{B}(t) / I_{0}(t)$, of experimental radiation-induced fluorescence decays at strong, $I_{B}(t)$, and nearly zero, $I_{0}(t)$, magnetic fields for rr-P3HT and rra-P3HT samples at $293 \mathrm{~K}$. Magnetic field values are indicated in the plot. Curves for rr-P3HT sample are shifted up by 0.15 . 


\section{Section S2}

\section{Backgrounds of the method of time-resolved magnetic field effects in recombination fluorescence and description of the model used to simulate TR MFE data for liquid state experiments}

The correlation between spin states of electrons filling a closed electronic shell in a molecule is kept after separating these electrons upon ionization of this molecule. In zero magnetic field (see Scheme S1), the spin states of a radical ion pair (RIP) are nearly degenerate thus facilitating equilibration of the populations of all these states to 0.25 due to hyperfine couplings (HFCs) as well as to phase paramagnetic relaxation (see, e.g., refs 1,2 in the reference list to this section below).
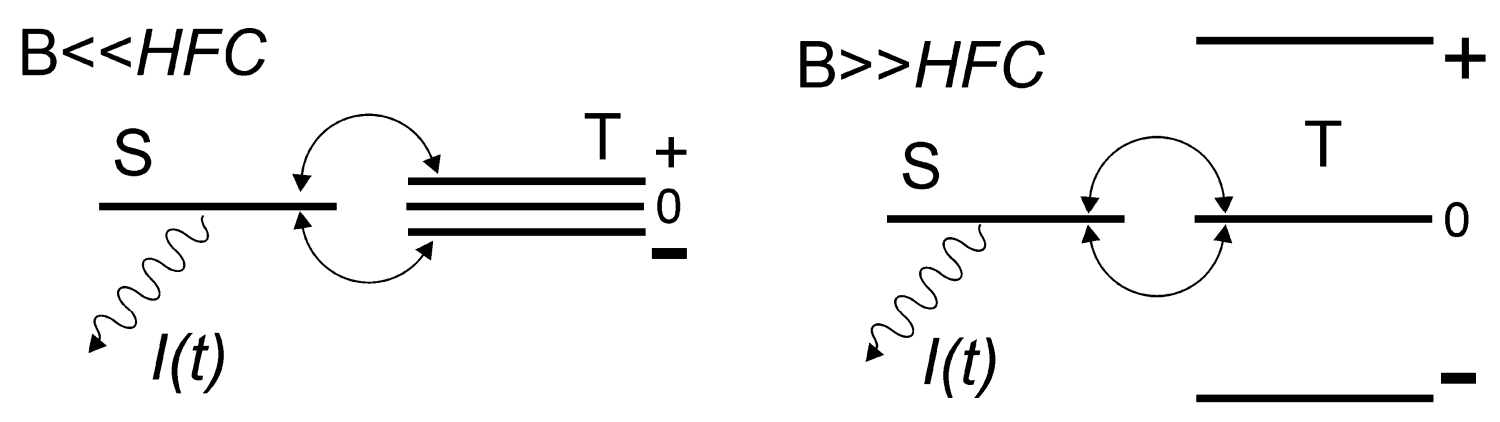

Scheme S1. Singlet (S) and triplets (T) spin sub-levels of a radical ion pair in nearly zero (left part) and in a strong magnetic field (right part) upon neglecting spin-spin interactions between the partners. Arrows show S-T mixing due to $H F C$ in the radical ions as well as phase paramagnetic relaxation. The wavy line is to emphasize that fluorescence can appear only due to the recombination of the RIP in its singlet state.

In a relatively strong magnetic field, these interactions as well as Zeeman interactions of the radicals composing the RIP, result in mixing of the singlet with, mainly, only one triplet state, corresponding to zero spin projection on the direction of the magnetic field. Therefore, in the absence of spin-lattice relaxation, the singlet spin state population for the singlet-born RIP after singlet-triplet mixing becomes of about 0.5. As in the experiments, the recombination fluorescence from the singlet spin state is observed turning on the strong magnetic field results in the fluorescence intensity grows with time by up to a factor of 2 . 
As the first approximation, the pulsed irradiation of a luminophore solution results in the decay of the recombination fluorescence intensity, $I(t)$, which is proportional to the recombination rate of the RIPs in the singlet spin state:

$$
I(t) \propto F(t) \cdot\left[\theta \rho_{S S}(t)+(1-\theta) / 4\right],
$$

where $\rho_{s s}(t)$ is the time dependence of the singlet state population in the subensemble of initially singlet-correlated RIPs. $\theta$ is a semiempirical parameter to take into account the fact that in the multiparticle radiation spur only a fraction of recombining RIPs is spin-correlated since some of them are composed of radical ions originating from the different primary ionization events. The second term in the brackets takes account of such spin-uncorrelated RIPs. The possibility of introducing the time-independent parameter $\theta$ was previously examined for low viscosity nonpolar solvents $[1,2]$. In particular, this implies that the recombination probability for a RIP is irreversible and does not depend on the RIP's spin state at other things equal.

In this approximation, complexities related to the experimental determining the RIPs' recombination kinetics, $F(t)$, can be avoided with studying the ratio of recombination fluorescence decays, $I_{B}(t)$ and $I_{0}(t)$, recorded at the strong and zero magnetic fields. This ratio is referred to as the time-resolved magnetic field effect (TR MFE):

$$
\frac{I_{B}(t)}{I_{0}(t)} \approx \frac{\theta \cdot \rho_{S S}^{B}(t)+(1-\theta) / 4}{\theta \cdot \rho_{S S}^{0}(t)+(1-\theta) / 4},
$$

where superscripts $B$ and 0 point to strong and zero external magnetic fields, respectively.

The RIP's singlet state population, $\rho_{s s}(t)$, for radical ions with isotropic g-tensors can be evaluated at the magnetic field $B$ as suggested previously (see, e.g. [1-3]):

$$
\begin{aligned}
& \rho_{s s}^{B}(t)=\frac{1}{4}+\frac{1}{4} \exp \left(-\frac{t}{T_{1}}\right)+\frac{1}{2} \exp \left(-\frac{t}{T_{2}}\right) \cos \left(\frac{\Delta g \beta B}{\hbar} \cdot \mathrm{t}\right) \cdot G_{c}^{B}(t) G_{a}^{B}(t) \\
& \rho_{s s}^{0}(t)=\frac{1}{4}+\frac{3}{4} \exp \left(-\frac{t}{T_{0}}\right) G_{c}^{0}(t) G_{a}^{0}(t)
\end{aligned}
$$

where $1 / \mathrm{T}_{1,2}=1 / T_{(a) 1,2}+1 / T_{(c) 1,2}$ are the sums of the longitudinal and phase relaxation rates of the RIP partners, and $T_{0}$ is the parameter to describe phase relaxation in a zero magnetic field in the same manner; $\Delta g$ denotes the difference between the $g$-values of the RIP partners; $\beta$ is the Bohr magneton. Subscripts " $a$ " and " $c$ " are to indicate that the parameters relate to radical anion (RA) and radical cation $(\mathrm{RC})$, respectively. 
In this work, the contribution of $H F C$ to the spin dynamics was calculated using the semiclassical approximation [3] of functions $G(t)$ as the following in the field units for $\sigma$,

$$
\begin{gathered}
G^{0}(t)=\frac{1}{3} \cdot\left[1+2 \cdot\left(1-(\gamma \sigma t)^{2}\right) \cdot \exp \left[-(\gamma \sigma t)^{2} / 2\right]\right], \\
\left.G^{B}(t)=\exp \left[-(\gamma \sigma t)^{2} / 2\right)\right],
\end{gathered}
$$

where $\sigma^{2}$ is the second momentum of the radical ion EPR spectrum $\left(\Delta H_{p p}=2 \sigma\right), \gamma=g \beta / \hbar$ is the electron gyromagnetic ratio.

Equations (S3) and (S4) were derived for the case of the absence of any radical ion reactions. If a radical ion participates in degenerate electron exchange, one should take into account a random change in the projections of nuclear spins in the radical. According to [3], for DEE involving, say, $\mathrm{RC}$, the $G^{B, 0}{ }_{c}(t)$ functions should be substituted for the functions $\Gamma^{B, 0}{ }_{c}(\mathrm{t})$ determined by the equation

$$
\Gamma_{c}(t)=\sum_{n=1}^{n=\infty} G_{c}^{(n)}(t)
$$

where $G_{c}^{(n)}(t)=\tau^{-1} \cdot \int_{0}^{t} G_{c}^{(1)}\left(t^{\prime}\right) \cdot G_{c}^{(n-1)}\left(t-t^{\prime}\right) d t^{\prime}$ is the term corresponding to the contribution of the random realization of (n-1) acts of self-exchange by the time $t, \tau$ is the mean time between electron jumps, and $G_{c}^{(1)}(t)=G_{c}(t) \cdot \exp (-t / \tau)$.

In the case when several parallel RIPs contribute to the recombination fluorescence intensity simultaneously then both the nominator and denominator in $\mathrm{Eq}(S 2)$ should include corresponding contributions of each pair. Note that the parameter $\theta$ should be the same for these contributions since this is determined by the primary radiation spur structure.

\section{References to the section S2}

1. Bagryansky, V.A.; Borovkov, V.I.; Molin, Y.N. Quantum beats in radical pairs. Russ. Chem. Rev. 2007, 76, 493-506.

2. Borovkov, V.; Stass, D.; Bagryansky, V.; Molin, Y. Study of Spin-Correlated Radical Ion Pairs in Irradiated Solutions by Optically Detected EPR and Related Techniques. In: 
Applications of EPR in Radiation Research, Ed. by Lund, A. and Shiotani, M. Eds.; Springer International Publishing: Cham, Switzerland, 2014; pp 629-663.

3. Schulten, K.; Wolynes, P. G. Semiclassical Description of Electron Spin Motion in Radicals Including the Effect of Electron Hopping. J. Chem. Phys. 1978, 68, 3292-3297.

\section{Section S3}

\section{Determination of the g-Factors of Radical Ions Used}

The TR MFE method only allows measuring the absolute value of the difference between the g-factors of recombining radical ions. Thus, to determine the g-factor value of a particular radical ion, which is a partner in the spin-correlated radical ion pair (RIP), the use of independent data about the g-factor of another partner is required. Typically, in EPR experiments these data were obtained under chemical generation of radical ions. For the generation of radical anions of aromatic compounds, solutions of $\mathrm{Na}$ or $\mathrm{K}$ are used.

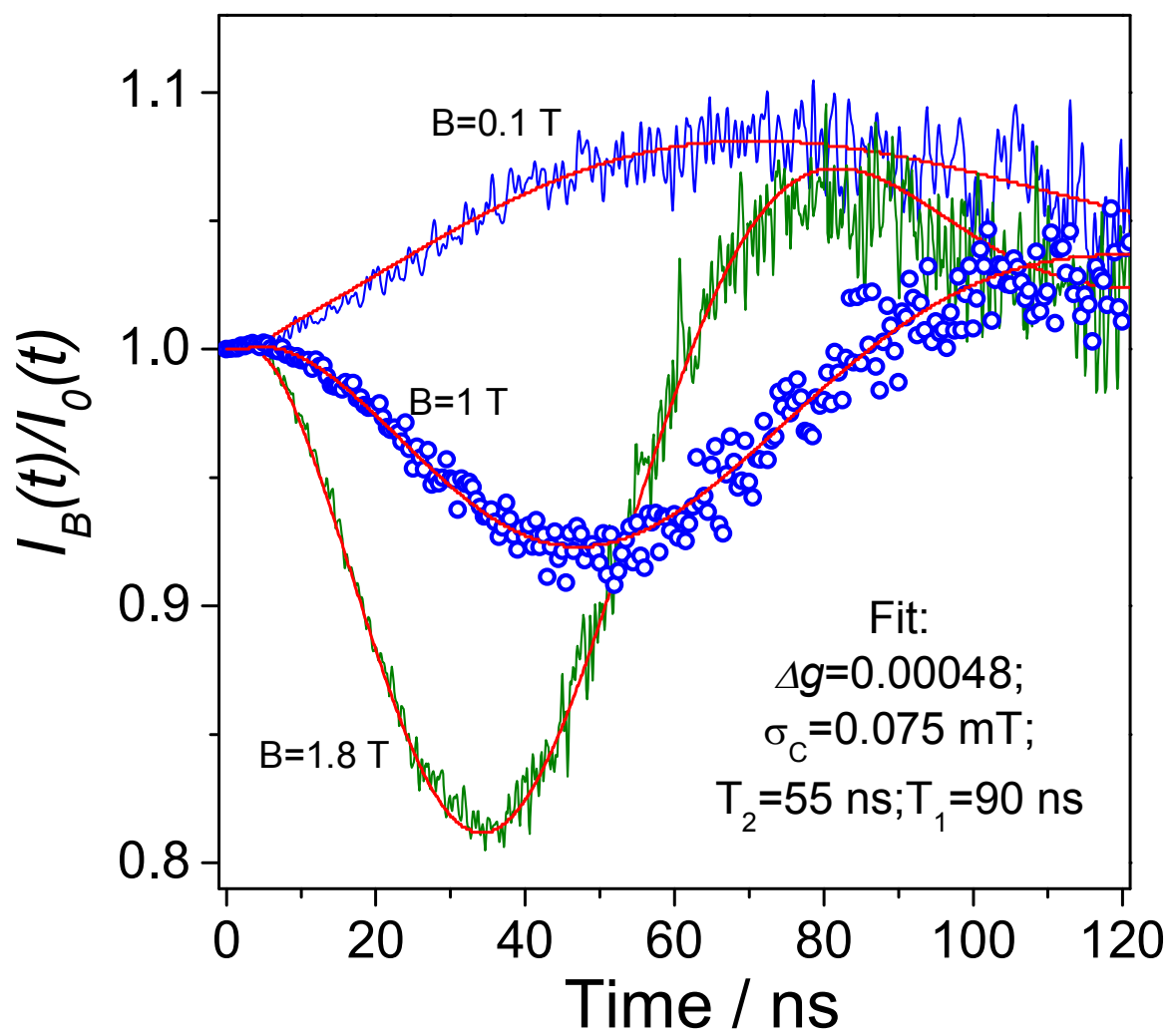

Figure S2. Ratios, $I_{B}(t) / I_{0}(t)$, of experimental radiation-induced fluorescence decays at strong and zero magnetic fields, respectively, for $0.3 \mathrm{mM}$ of $p$-TP in methanol at $293 \mathrm{~K}$. Magnetic field values and simulation parameters are indicated in the plot. 
For the radical cations, strong oxidizing agents, like trifluoroacetic acid, are applied. Consequently, in these experiments, the measured g-values can be changed due to "ion-pairing" effect. For the purpose of the present study, values of the g-factors of isolated para-terphenyl $(p \mathrm{TP})$ radical cation and radical anions were examined in conditions of radiation experiments performed in liquid solutions without additional chemical agents.

Simulation was performed using eqs. S2-S6. The $\sigma$-values for $p$-TP radical cation, $0.0075 \mathrm{mT}$, and radical anion, $0.069 \mathrm{mT}$, were calculated using literature values of HFC constant in protiated radical ions $[4,5]$. para-Terphenyl- $d_{14}(98 \%$, Aldrich) was used as received.

First, the $g$-factor of $p$-TP radical cation was determined in the solution in absolute methanol. In this case, observed radiation-induced fluorescence appears due to recombination of this radical cation and solvated electron, the $g$-factor of which in methanol (2.00205) was carefully measured by EPR spectroscopy [6]. In Fig. 2S, the experimental and calculated TR MFE curves for $0.3 \mathrm{mM} p \mathrm{TP}-\mathrm{d}_{14}$ solution in absolute methanol at $293 \mathrm{~K}$ are shown.

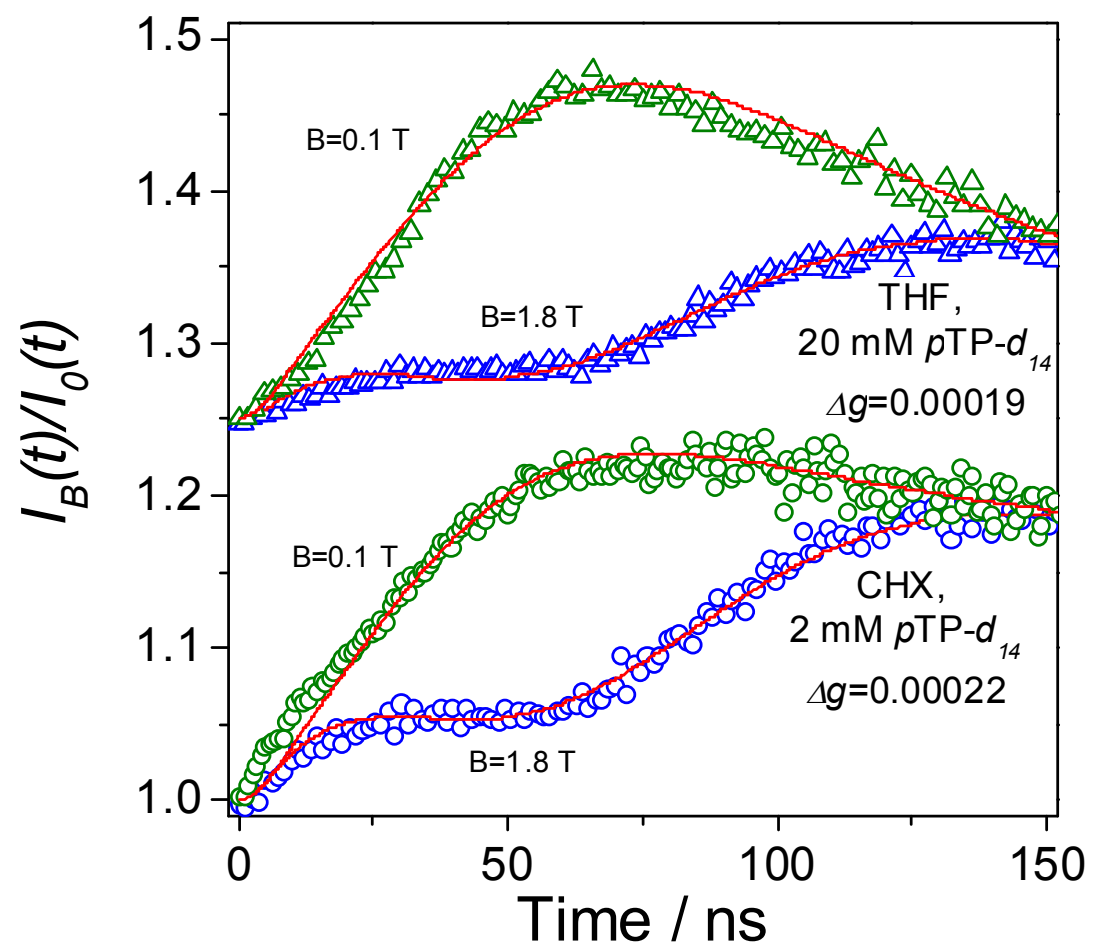

Figure S3. Ratios, $I_{B}(t) / I_{0}(t)$, of the radiation-induced fluorescence decays at $\mathrm{B}=0.1 \mathrm{~T}$ (green symbols) or $\mathrm{B}=1.8 \mathrm{~T}$ (blue symbols) and zero magnetic fields, respectively, at $293 \mathrm{~K}$ for solutions of $p \mathrm{TP}-d_{14}$ in the concentration of $20 \mathrm{mM}$ in tetrahydrofuran (triangles, the curves are shifted up by 0.25 ) and of $2 \mathrm{mM}$ in cyclohexane (circles). Red lines show the calculated TR MFE curves at $\Delta g$ values of recombining radical ions as large as $1.9 \cdot 10^{-4}$ (THF) and $2.2 \cdot 10^{-4}(\mathrm{CHX})$. Phase relaxation times, $T_{2}$ and $T_{0}$, were in the range of 50-70 ns. 
The calculated curves were obtained using eqs. S2-S6. Note that in this case, $\mathrm{T}_{1}$ value, most probably, is not a real spin-lattice relaxation time but it reflects a decrease in the contribution of recombination fluorescence against of the background luminescence with time.

The optimal fits were obtained at the difference between g-factors of the RIP partners equaled to $\Delta g_{a v}=0.00048( \pm 1)$ that leads to the g-factor of $p \mathrm{TP}$ radical cation as large as 2.00253 . This is in a good agreement with the value of 2.0025 as reported in EPR study, which exploited photolysis of a solution of terphenyl in trifluoroacetic acid containing mercury(II) trifluoroacetate [4].

Further, the $\Delta g$ value for the pair $p-\mathrm{TP}^{+\bullet} / p-\mathrm{TP}^{-\bullet}$ was determined (Fig. S3). The experimental conditions were chosen in such a way to create rapidly spin-correlated pairs of interest owing to a high concentration (tetrahydrofuran) or a very fast scavenging of primary charge carriers by aromatic molecules (cyclohexane). The simulation performed for both solvents suggested that the $g$-factor $p$ TP radical anion falls into the range of 2.0027-2.0028 that is in accordance with data from ref [5].

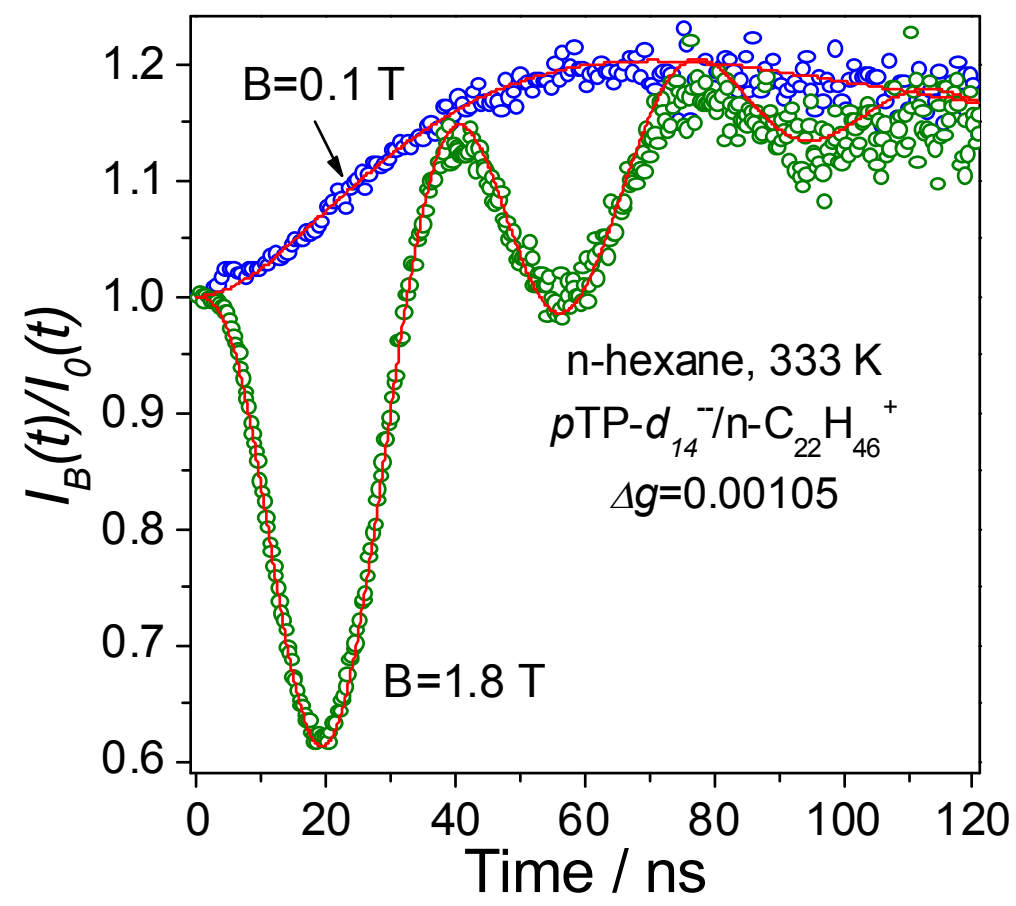

Figure S4. Experimental and calculated ratios (TR MFE curves), $I_{B}(t) / I_{0}(t)$, of delayed fluorescence intensity decays recorded at a high and nearly zero magnetic fields, respectively, for solution of $0.1 \mathrm{mM}$ $p$ TP- $\mathrm{d}_{14}$ and $30 \mathrm{mM}$ of $n$-docosane in $n$-hexane at $333 \mathrm{~K}$. The calculated curves were obtained at $\Delta g_{a v}=0.00105( \pm 3)$, phase relaxation times $T_{2}=T_{0}=55 \mathrm{~ns}$ independently of the magnetic field strength. 
The value of the g-factor of $p \mathrm{TP}^{-\bullet}$ was used to determine the g-factor of $n$-docosane radical cation (see Fig. S4). In $n$-hexane solutions containing $n$-docosane and very small amount of $p$ $\mathrm{TP}$, docosane molecules scavenge only solvent holes while excess electrons are scavenged by $p$ TP molecules. Therefore, TR MFE curves presented in Fig. S4, reflect the spin dynamics in the spin-correlated pairs $n-\mathrm{C}_{22} \mathrm{H}_{46}{ }^{+\bullet} / p \mathrm{TP}^{-\bullet}$. As in the cases of other $n$-alkanes [7], the degenerate electron exchange involving the radical cation should be taken into account using eq. S7. The best fits at different concentrations and temperatures (not shown here) were obtained assuming that the docosane radical cation in the absence of the exchange has $\sigma \approx 0.15 \mathrm{mT}$ and the characteristic time between electron jumps at $333 \mathrm{~K}$ was $\tau \approx 10 \mathrm{~ns}$.

According to the simulations, the difference between the g-values of these species is close to 0.00105. The relative error for this value is rather small since the well pronounced oscillations in the TR MFE curves are observed (Fig. S4). In such a case, the error was determined mainly by the relative accuracy of the set-up of a strong magnetic field that certainly did not exceed $1 \%$. As the g-values of alkane radical cations are higher as compared to radical ions of aromatic molecules then the g-value of docosane radical cation is about 2.0038. The estimated error of this value is less than 0.0001 .

Note that no magnetic field dependence of the phase paramagnetic relaxation rate was found for the pair containing $n-\mathrm{C}_{22} \mathrm{H}_{46}{ }^{+\bullet}$. The $T_{2}$ values used in the simulations described in this section are rather close to each other. This could suggest (see Eq. S4) that the phase relaxation rate for either radical ion of $p$-TP or docosane radical cation is of about $\sim 10^{-2} \mathrm{~ns}^{-1}$ that corresponds to homogeneous broadening of about $\sim 0.1 \mathrm{mT}$ for each of the radicals. It is not clear at this stage, why such a broadening is similar for such different particles. It is probably that this broadening is partially determined by dephasing process, which occurs in primary solvent holes or in products of their fast transformation before scavenging these radical cations by solute molecules.

\section{References to the section S3}

4. Courtneidge, J. L.; Davies, A. G.; McGuchan, D. C. The Electron Spin Resonance Spectra of the Radical Cations of p-Terphenyl, Triphenylene and Triptycene. Recl. Trav. Chim. Pays-Bas. 1988, 107, 190-196.

5. Berndt, A.; Jones, M. T.; Lehnig, M.; Lunazzi, L.; Placucci, G.; Stegmann, H. B.; Ulmschneider, K. B. Landolt-Bőrnstein. Numerical Data and Functional Relationships in 
Science and Technology. Organic Anion Radicals; New Series, Subvolume 9d1; SpringerVerlag: Berlin, Germany, 1980; p 830.

6. Shiraishi, H.; Ishigure, K.; Morokuma, K. An ESR study on Solvated Electrons in Water and Alcohols: Difference in the $g$-Factor and Related Analysis of the Electronic State by MO Calculation. J. Chem. Phys. 1988, 88, 4637-4649.

7. Borovkov,V.I .; Gritsan, N. P.; Yeletskikh, I. V.; Bagryansky, V. A.; Molin, Y. N. Degenerate Electron Exchange Reaction of $n$-Alkane Radical Cations in Solution. J. Phys. Chem. A 2006, $110,12752-12759$.

\section{Section S4}

Results of quantum chemical calculations:

Structure, single occupied molecular orbital (SOMO), and the $g$-tensor components for radical anions and radical cation of methyl-thiophene (3-MT) olygomers (axes' directions are shown in Figure S9)

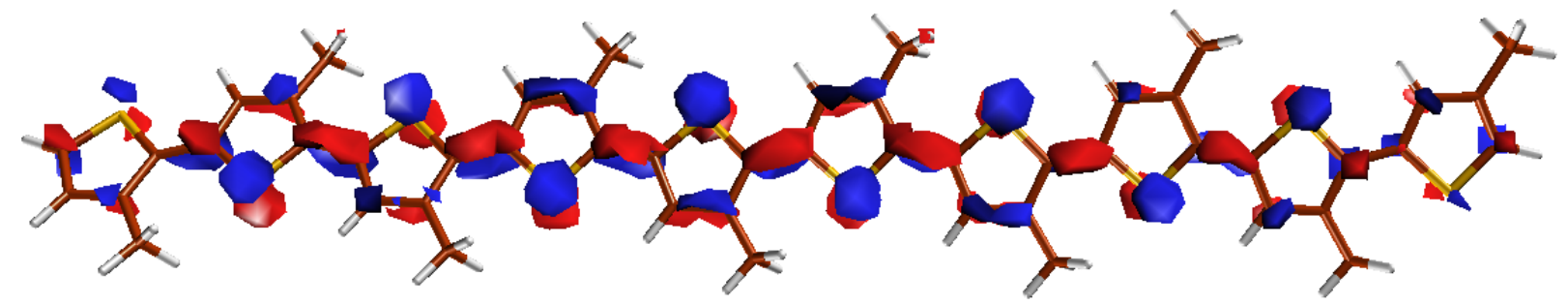

$\mathrm{g}=(2.0023 ; 2.0024 ; 2.0080) \quad g_{a v}=2.0042$

Figure S5. $\quad(3-\mathrm{MT})_{10^{-}}$, regioregular, SOMO (SPACE=0.02, MOLDEN program [8] was used)

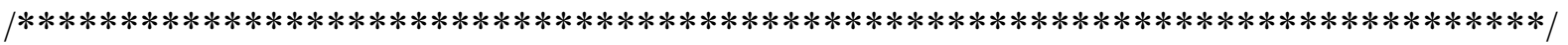

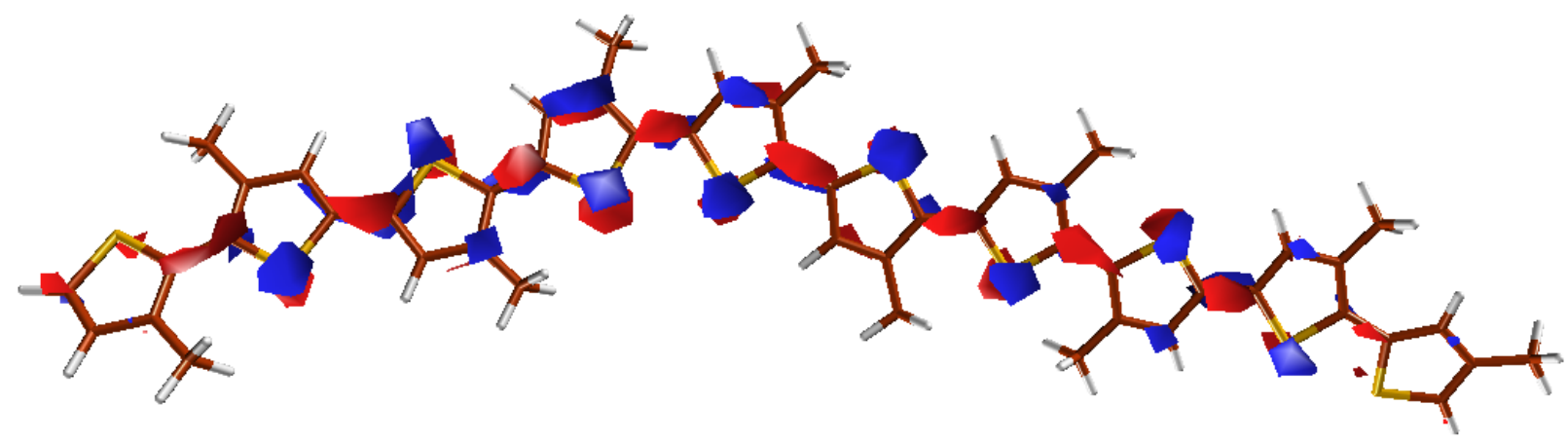

$\mathrm{g}=(2.00216 ; 2.0024 ; 2.0072) \quad g_{a v}=2.0041$

Figure S6. $\quad(3-\mathrm{MT})_{10}{ }^{\bullet}$, regiorandom, SOMO (SPACE=0.02) 


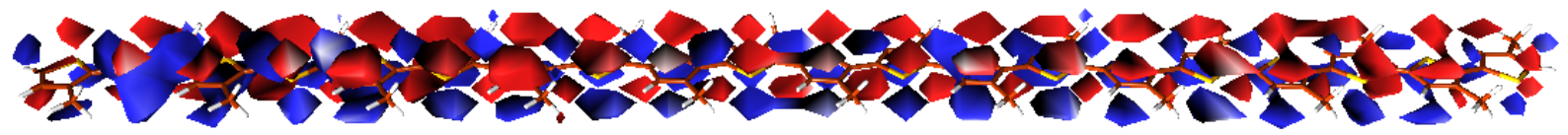

$\mathrm{g}=(2.0022 ; 2.0024 ; 2.0082) \quad g_{a v}=2.0043$

Figure S7. $\quad(3-\mathrm{MT})_{20}{ }^{-\bullet}$, regioregular, linear conformation, SOMO (SPACE=0.003)

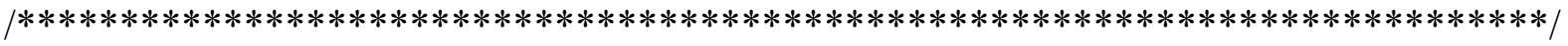

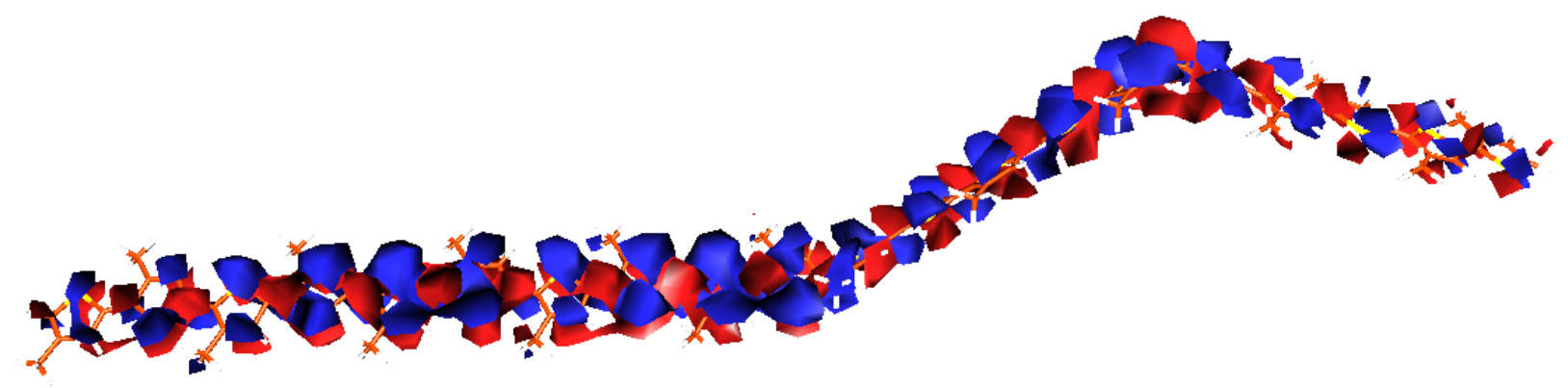

$g=(2.0029 ; 2.0039 ; 2.0075) \quad g_{a v}=2.0047$

Figure S8. $\quad(3-\mathrm{MT})_{20}{ }^{\bullet}$, regioregular, broken conformation, SOMO (SPACE=0.005)

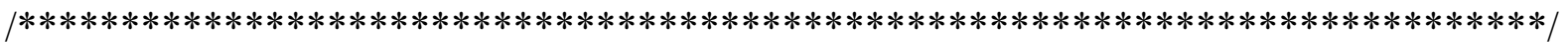
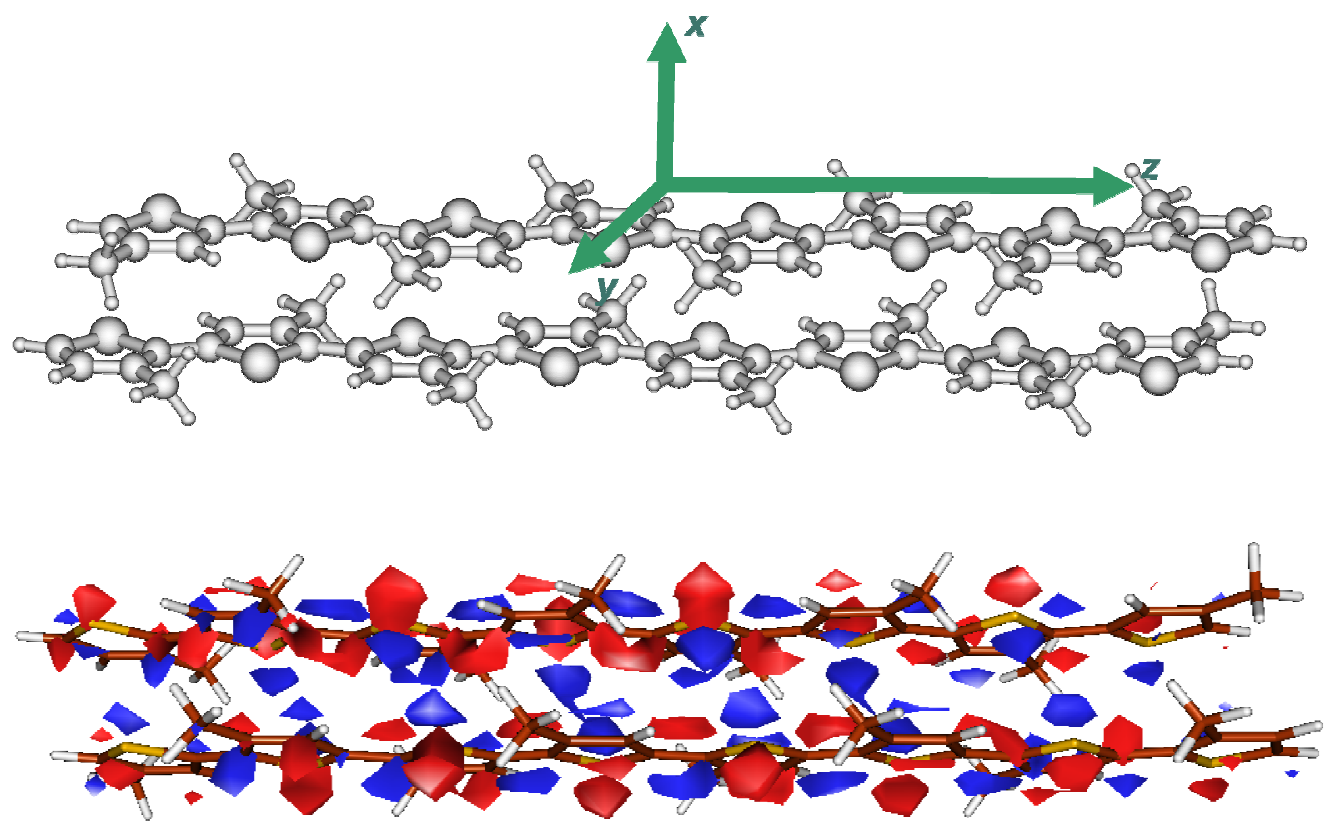

$\mathrm{g}=(2.00236 ; 2.0026 ; 2.0087)$

$$
g_{a v}=2.0046
$$

Figure S9. $\quad\left[(3-\mathrm{MT})_{8}\right]_{2}{ }^{-\bullet}$, regioregular dimeric structure $(8+8)$ chains, SOMO (SPACE $\left.=0.02\right)$ 


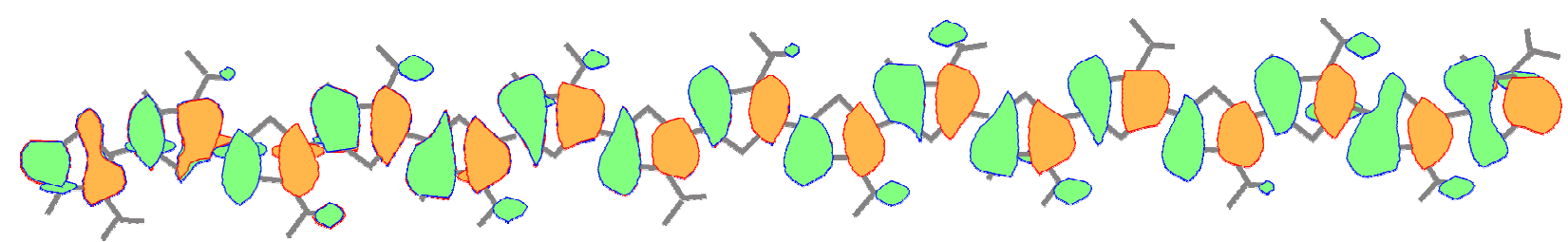

$\mathrm{g}=(2.0023 ; 2.0024 ; 2.0014)$

$$
g_{a v}=2.0020
$$

Figure S9. $\quad\left[(3-\mathrm{MT})_{16}\right]^{+\bullet}$, regioregular, SOMO $(\mathrm{SPACE}=0.004)$

\section{Reference to the section S4.}

8. Schaftenaar, G.; Noordik, J. H. Molden: a pre- and post-Processing Program for Molecular and Electronic Structures. J. Comput.-Aided Mol. Des. 2000, 14, 123-134. 